\title{
A DRIVERLESS VEHICLE DEMONSTRATION ON MOTORWAYS AND IN URBAN ENVIRONMENTS
}

\author{
Jorge Godoy ${ }^{1}$, Joshué Pérez ${ }^{2}$, Enrique Onieva ${ }^{3}$, Jorge Villagrá ${ }^{4}$, \\ Vicente Milanés ${ }^{5}$, Rodolfo Haber ${ }^{6}$ \\ ${ }^{1,4,6}$ AUTOPIA Program, Centre for Automation and Robotics (CAR), Madrid, Spain \\ ${ }^{2,5}$ IMARA team at INRIA Research Center, Paris, France \\ ${ }^{3}$ University of Deusto, Bilbao, Spain
}

Submitted 24 July 2014; resubmitted 19 September 2014; accepted 19 December 2014; first published online 28 January 2015

\begin{abstract}
The constant growth of the number of vehicles in today's world demands improvements in the safety and efficiency of roads and road use. This can be in part satisfied by the implementation of autonomous driving systems because of their greater precision than human drivers in controlling a vehicle. As result, the capacity of the roads would be increased by reducing the spacing between vehicles. Moreover, greener driving modes could be applied so that the fuel consumption, and therefore carbon emissions, would be reduced. This paper presents the results obtained by the AUTOPIA program during a public demonstration performed in June 2012. This driverless experiment consisted of a 100-kilometre route around Madrid (Spain), including both urban and motorway environments. A first vehicle acting as leader and manually driven - transmitted its relevant information - i.e., position and speed - through an 802.11p communication link to a second vehicle, which tracked the leader's trajectory and speed while maintaining a safe distance. The results were encouraging, and showed the viability of the AUTOPIA approach.
\end{abstract}

Keywords: intelligent transportation systems (ITS); road vehicles; vehicle-to-vehicle (V2V) communication; cooperative adaptive cruise control (CACC); artificial intelligence.

\section{Introduction}

Although fully autonomous vehicles are still out of reach of the general public, a start has been made through the inclusion of several driving-aid systems in today's cars. The development of these kinds of systems is mainly motivated by the increasing number of vehicles on the roads, which demands improvements in terms of safety and efficiency. According to the information provided by Eurostat (Eurostat 2012), it is estimated that the number of passenger cars increased by around 12\% from 2001 to 2009 just in the European Union. A feasible solution to cope with this growth is to increase the capacity of present roads by reducing the spacing between cars (Shladover 2009). However, in order to achieve this goal it would be necessary to develop control systems with a greater degree of precision than human drivers. In this sense, platooning - i.e., forming a group of vehicles traveling closely spaced - is one of the most promising technologies.

Several of the first works related to the development and implementation of platooning were carried out in the Partners for Advanced Transportation TecHnology (PATH) program - one of the pioneer developers in the field of Intelligent Transportation Systems (ITS) in the mid-90's. The results showed that this solution provides extra benefits in terms of both safety, due to the very low relative speed of the vehicles, and fuel consumption, due to the reduction of aerodynamic drag on the following vehicles (Varaiya 1993). In August 1997, the program held a demonstration in San Diego (USA) with the goal of testing the feasibility of the system. During the platoon demonstration, 8 vehicles successfully travelled together, maintaining highly precise spacing, by using a control system based on radar measurements and radio communication.

The considerable recent advances in communication technologies have opened up a new framework for ITS research on innovative approaches based on vehicle-to-vehicle $(\mathrm{V} 2 \mathrm{~V})$ and vehicle-to-infrastructure (V2I) links. Several manufacturers have teamed up into a communication consortium known as Car-toCar, whose main goal is to contribute to the develop- 
ment and release of an open communication standard for vehicular environments (Car2Car 2012). One of the works in this research line is the SARTRE project, which finished late last year. This project was aimed at examining the possible issues involved in allowing platoons to operate on public motorways without any infrastructure modification (Bergenhem et al. 2010; SARTRE 2012). Another significant work was the Grand Cooperative Driving Challenge (GCDC) held in Helmond (Netherlands) in May 2011 (GCDC 2012). During this competition, nine international teams had the opportunity to test and compare their cooperative applications for urban and motorway scenarios (Van Nunen et al. 2012). The common factor among the different platforms was the communication system. This was based on an iteration protocol especially developed for the challenge (De Jongh 2011).

In parallel with the research on applications for motorway environments, there have also been several works carried out in urban contexts. Indeed, the urban environments represent a more complex challenge because of the multiple special features they present - e.g., intersections, pedestrian crossings, roundabouts, and so on. For this reason, most of the applications developed up to now have been semi-autonomous driving aid systems. One such solution is the City Safety system, developed and commercialized by Volvo, which is able to stop the car - at speeds lower than $15 \mathrm{~km} / \mathrm{h}$ - when an obstacle is detected in front of it (Distner et al. 2009).

The present contribution describes a control approach for driverless vehicles on motorways and in urban environments. The system is based on V2V communication among the cars, allowing a leading vehicle to generate online a high-precision digital map of the road. Using this information, a trailing vehicle is able to follow the same route as the leader. Moreover, the implementation of a Cooperative Adaptive Cruise Control (CACC) system allows the trailing vehicle to track the leader's speed while maintaining a safe time gap between the two. In order to test the performance of the system, two vehicles of the AUTOPIA program - two Citroën C3's were equipped with the system, and a public demonstration was performed with good results.

The rest of the paper is structured as follows. Section 1 presents a brief description of the AUTOPIA program and the control architecture implemented in its vehicles. An introduction to the problem of controlling autonomous vehicles is presented in Section 2. Section 3 explains the trajectory generation process. The fuzzy logic controllers for the two environments are described in Section 4. The results from the public demonstration are presented in Section 5. Finally, the last section presents some concluding remarks and outlines future work.

\section{The AUTOPIA Program}

The AUTOPIA Program is a research group belonging to the Centre for Automation and Robotics (CAR) of the Spanish National Research Council (CSIC) and the Technical University of Madrid (UPM). With more than
15 years' experience, AUTOPIA is the pioneer team in vehicle automation in Spain. Starting with the automation of two electric vans, this program has expanded its research line to the development of intelligent driving aid systems and the implementation of cooperative manoeuvres based on fully autonomous vehicles and wireless communications (Milanés et al. 2012).

The team now has a fleet of 5 fully automated vehicles. Each vehicle is equipped with a control system based on a highly modular architecture, implemented in both software and hardware. The AUTOPIA system is divided into four main stages: Perception, Actuation, Decision, and General I/O. Each of these stages is subdivided into smaller modules with more specific tasks (Fig. 1). The backbone of the Perception stage is the Differential GPS unit, which, combined with an Inertial Measurement Unit (IMU) and the CAN (Controller Area Network) bus data, provides high precision measurements of the vehicle's attitude (Milanés et al. 2008). The Actuation stage comprises several low-level modules that allow the on-board PC to control the vehicle's actuators (steering wheel, brake, and throttle). Nevertheless, the key for autonomous driving is the Decision stage, in which a combination of fuzzy controllers imitates the behaviour of a human driver. These controllers have been developed and tested for different scenarios such as roundabouts (Pérez et al. 2011a), overtaking (Naranjo et al. 2008), merging (Milanés et al. 2011a) and emergency scenarios (Milanés et al. 2011b). For path planning, the Decision stage implements two modules:

- the mission module, in which the main list of goals for the vehicle is managed;

- a planner module, which generates an appropriate reference for the vehicle's trajectory based on the mission data.

The last stage, General I/O, performs auxiliary tasks for the previous three. It comprises several I/O modules for digital and analogue signals, allowing the PC to interact with such vehicle components as lights, gear, and horn. Two communication modules are also included in this stage:

- a Wi-Fi module, used in the first manoeuvres based on V2V communications;

- a communication gateway developed for the GCDC and based on the IEEE 802.11p standard (Van Nunen et al. 2012).

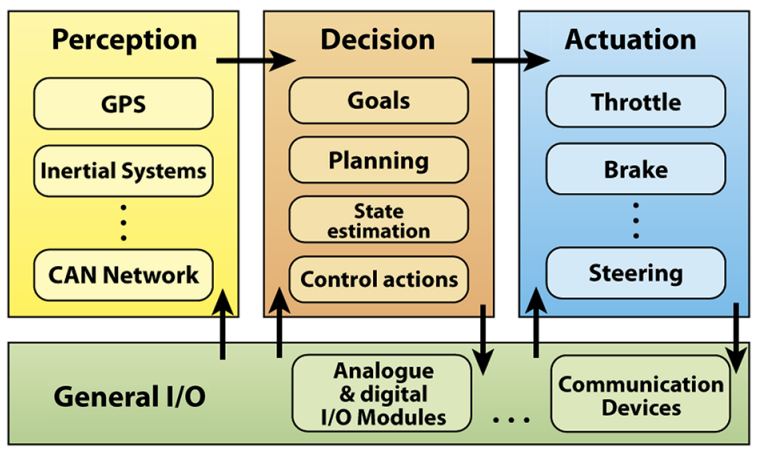

Fig. 1. Scheme of AUTOPIA's control architecture 
The major constraint of the AUTOPIA system is associated to the multipath problem and the GPS localization. In previous works authors have shown different methods for dead reckoning, successfully overcoming up to 2 minutes of GPS outages (Milanés et al. 2008; Godoy et al. 2012). Although this solve the problem for short gaps (e.g. bridges, small tunnel, traffic signals), it is still an issue when dealing with longer outages.

\section{Understanding the Scenario}

The task of controlling a vehicle autonomously can be divided into two sections: longitudinal control, in which only the throttle and brake are considered; and lateral control, covering actuation on the steering wheel. Nevertheless, this division does not mean that actions taken in one section are totally unrelated to the actions in the other. Of the two sections, the task of lateral control is more complex than that of longitudinal control.

There are two main challenges present in the development of a suitable steering wheel control system:

- providing a reliable road reference;

- development of a controller capable of following the reference safely.

A common solution to the first problem is the implementation in vehicles of high precision cartography and localization systems, which can be achieved in different ways. For example, the PATH program implements a localization system based on magnetic markers installed along the roadway in specific patterns. These patterns allow road information to be transmitted to the vehicles - e.g., road curvature, current milepost, etc. (Tan, Bougler 2001). However, that this option requires modifications to be made to the roadway is a major drawback. Another option is the implementation of localization systems based on Differential GPS units installed in the vehicles. These are able to provide vehicle localization information without needing changes to be made to the infrastructure. Nevertheless, this system is only appropriate when the route is known or a high precision digital map has been defined previously. Both options are susceptible to failure due to road modifications not included in the cartography - e.g., road works.

An alternative to this strategy is to tackle the problem using simultaneous mapping and localization techniques (SLAM) (Durrant-Whyte, Bailey 2006) such as those based on computer vision (Lategahn et al. 2011; Rituerto et al. 2010) or LIght Detection And Ranging (LIDAR) (Levinson et al. 2011; Paz et al. 2008), and which have already given promising results. However, the main drawback of most of these approaches is their dependence on high performance sensor systems, which makes them unaffordable for mass-produced vehicles.

Bearing this in mind, our proposed solution is based on the online generation of routes. The goal is to use the information transmitted by a leading vehicle to generate a suitable route for trailing vehicles in real time. It is assumed that the leading vehicle is equipped with the same control architecture, or at least with the same perception and communication modules. This solution allows the trailing vehicles to follow a leader over any route without having any previous knowledge about it. Moreover, the leading vehicle can be autonomously or manually driven without this implying any major changes for the trailing vehicles. This approach is similar to that used in the VIAC experiment, in which a group of vehicles drove about $8300 \mathrm{~km}$ in autonomous mode without previous knowledge of the route (Bertozzi et al. 2013).

With respect to longitudinal control, the goal of the trailing vehicle is to track the speed of the leader while also keeping a safe distance between the two vehicles. To this end, the implementation of a CACC system based on V2V communications is the best option. This system will manage throttle and brake actions in accordance with both the latest information received from the leader and the current state of the trailing vehicle.

\section{Trajectory Generation}

As was mentioned in Section 1, the architecture includes two key modules for path planning: the mission and the planner modules. The leading vehicle informs about its state continuously through the communication system. Every transmitted message includes speed and position information. Based on the data received, the trailing vehicle is able to keep a real-time trace of the trajectory followed by the leader. In particular, the position data is stored by the mission module as checkpoints for the car. In parallel, the planner module processes the mission data and generates a suitable reference path for the trailing vehicle.

For the inclusion of checkpoints in the mission list, the system addresses three additional issues:

- when it is initialized, the system sets the current position of the trailing vehicle as the first checkpoint in the mission module. The planner is then able to start generating the route as soon as the first message from the leading vehicle is received since it requires a minimum of two points to run;

- to reduce the number of checkpoints added, a minimum distance $d_{\min }$ is required between the last added point and a new one. If a closer position is received, it is discarded automatically from the route generation. The value of $d_{\min }$ is set to be an appropriate trade-off between restricting the number of checkpoints to process and maintaining resemblance of the trace with the real trajectory of the leader;

- a lower speed threshold is defined in the sense that no points are included in the mission list if the speed of the leading vehicle is less than $1 \mathrm{~km} / \mathrm{h}$.

An example trajectory generation is shown in Fig. 2. The path travelled by the leading vehicle is represented by the grey line. The black dots show the final points created by the planner module to define the reference trajectory. The data was collected during a real-world experiment performed in an urban area, with speeds between 10 and $30 \mathrm{~km} / \mathrm{h}$. In the inset, the checkpoints used 


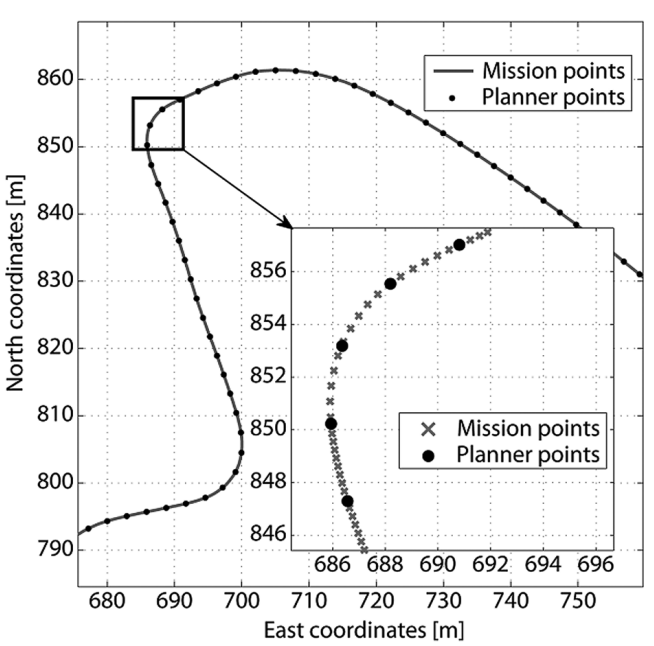

Fig. 2. Example of the trajectory generation

by the mission module to trace the leader are shown as grey $\times$ 's over a zoomed-in area of the trajectory. These points correspond to the data received from the leader after considering the constraints defined above for the inclusion of new points. One can appreciate in the graph that, regardless of the number of mission points present in any segment, the planner always extrapolates the data in order to have equally separated points.

\section{Vehicle Control}

From its beginnings with the control of a single vehicle in a closed environment to the management of vehicles arriving at an intersection, the approach followed by the AUTOPIA program has been the same: application of artificial intelligence techniques to emulate human behaviour when driving. After having combined different techniques such as neural networks (Pérez et al. 2010) or genetic algorithms (Onieva et al. 2012), the group now considers the application of fuzzy logic as the best option to transform drivers' knowledge into a controller capable of driving a car autonomously in different scenarios.

Fuzzy logic is based on the theory of fuzzy sets presented in (Zadeh 1965). In contrast with classical set theory, where the membership of an element in a set is represented by a binary value, i.e., belonging or not belonging to the set, fuzzy set theory defines this relationship through a continuous value in the range $[0,1]$, with 1 being the maximum degree of membership. Moreover, the association of linguistic variables with the definition of each set allows the application of reasoning which is more like human reasoning (Zadeh 1996).

For the execution of fuzzy controllers in vehicles, a specially designed tool denominated ORBEX (García Rosa, De Pedro Lucio 2000) was used. It was developed by members of the CAR as a solution for real-time applications. ORBEX codifies the input variables using trapezoidal membership functions, which are defined using only four values - i.e., $F(a, b, c, d)$. The output variables are codified by discrete values representing singleton functions. This is done with the goal of reducing the time needed to compute the controller outputs (Sugeno 1999). Through a configuration file, the user is able to define the controller rules using text strings of the kind: IF input input_label THEN output output_label.

\subsection{Longitudinal Control}

Several vehicle manufacturers currently provide various options of Adaptive Cruise Control (ACC) systems for their cars. Nevertheless, most of them are based on camera, radar, or laser perception. With respect to the subset of communications-based systems (CACC), there have been several experimental approaches in this line. For example, in (Naranjo et al. 2007) a fuzzy-based CACC system for urban scenarios is presented. Moreover, the system is also able to manage Stop\&Go scenarios in which the car may be stopped for a short time.

Vehicle dynamics at low speeds are still poorly understood, and such variables as road friction, weight of occupants, or slopped and banked roads may affect the performance of an ACC controller to a greater extent than in a high-speed scenario. This hinders the development of a controller able to work equally well in both scenarios. To resolve this issue, a fuzzy controller was developed which has an online learning capability.

This proposal is an evolution of the system previously presented by AUTOPIA in (Onieva et al. 2013). There, a valid method for the real-time evolution of a fuzzy controller for Cruise Control (CC) was described. Starting from a simple configuration, the system was able to adapt the consequent of the rules and modify the Membership Functions (MFs) in accordance with the changing and uncertain vehicle dynamics and to road disturbances.

Two variables were defined as inputs for the fuzzy controller:

- the relative speed $r s$ between the leading and the trailing vehicles, expressed in $\mathrm{km} / \mathrm{h}$;

- the difference de between the distance reference and the real separation of the vehicles, expressed in metres.

For safety reasons, the system estimates the distance reference $d_{r}$ as a value proportional to the leader's speed:

$$
d_{r}=d_{0}+0.6 v_{l}
$$

where: $d_{0}$ being the minimum distance allowed between the vehicles; $v_{l}$ the leader's speed in $\mathrm{m} / \mathrm{s}$. The value $d_{0}$ was set at 10 metres since this was the safety distance required for the GCDC competition (Kwakkernaat 2011). The constant 0.6 in the equation is the time gap - in seconds - between the vehicles. It was set in accordance with usual values found in the literature for CACC systems, guaranteeing crash avoidance under emergency conditions and taking into consideration the acceptance by drivers from the general public (Nowakowski et al. 2010; Milanés et al. 2014). The distance between cars is measured as the distance between their GPS antennas.

To manage both throttle and brake, the controller uses a single output defined in the interval $[-1,1]$. 
Positive values represent actions on the throttle with no brake actions and negative values vice versa.

The following configuration was used as the base controller for learning:

- each input is codified by four MFs;

- a rule is defined for each possible input combination, so that there are sixteen rules in total;

- for each rule, a unique output singleton is defined. This is necessary for the adjustment of one rule not to affect another one.

An initial adjustment of the controller was necessary since no modification to the input MFs is allowed during learning. Because of this, a set of experiments was conducted at different speeds between 10 and $90 \mathrm{~km} / \mathrm{h}$. At the end of each test, the results were analysed and the controller adjusted manually. The process was repeated until a configuration capable of keeping the distance error below 2 metres in that speed range was obtained. The final MF configuration is shown in Fig. 3. The values set for each output singleton are listed in Table.

Starting from this configuration, the learning algorithm is responsible for adjusting the output singletons in real time in accordance with the evolution of the control variables. In each cycle, the system evaluates the performance of the current configuration and determines the reward $\gamma$ to be applied to the activated singletons. Fig. 4 shows the reward obtained in accordance with the two input values. One sees in the figure that the designation of the reward seems natural. For example, if the vehicles are closer than the reference, but the trailing one is slower than the leader, the singletons are not modified. However, if the trailing vehicle starts to go faster than the leader while the distance is too short, the singletons are reduced.
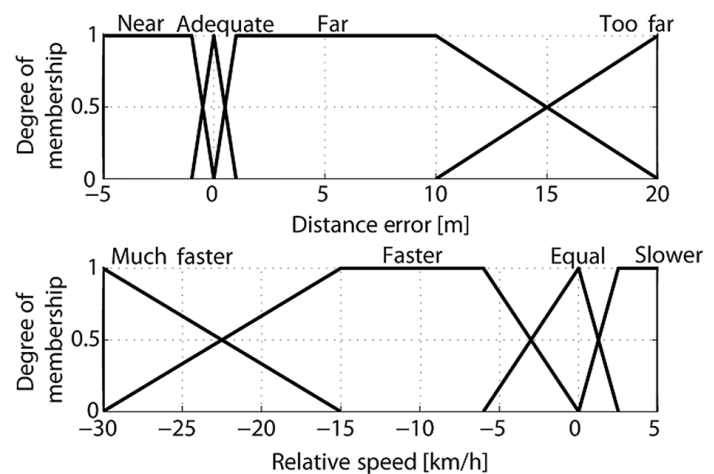

Fig. 3. Membership functions for CACC controller inputs

Table. Initial singleton values

\begin{tabular}{lcccc}
\hline \multirow{2}{*}{$\begin{array}{c}\text { Distance } \\
\text { error }\end{array}$} & \multicolumn{5}{c}{ Relative speed } \\
\cline { 2 - 5 } & Slower & Equal & Faster & Much faster \\
\hline Near & 0 & -0.6 & -1 & -1 \\
\hline Adequate & 0.25 & 0 & -0.6 & -1 \\
\hline Far & 0.8 & 0.5 & 0 & -1 \\
\hline Too far & 0.8 & 0.8 & 0.8 & 0.215 \\
\hline
\end{tabular}

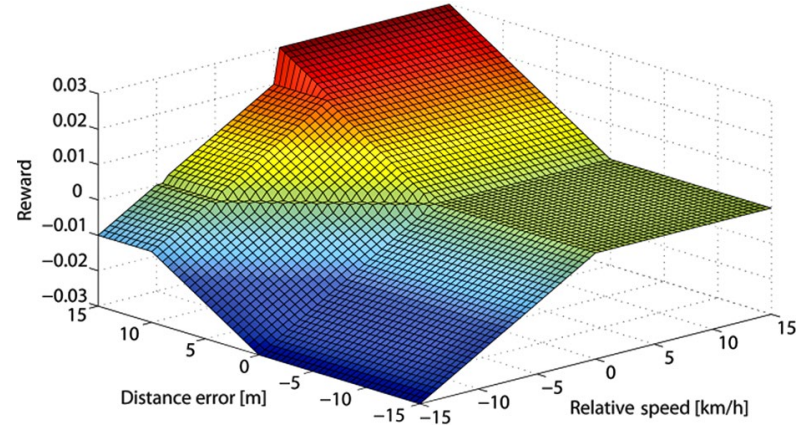

Fig. 4. Reward according to the input values

Whichever the case, only the singletons corresponding to the triggered rules are modified. The modification is proportional to the activation of the rules:

$$
R_{i}(k)=R_{i}(k-1)+\mu_{i}(k-1) \gamma(\operatorname{de}(k), r s(k)),
$$

where: $R_{i}$ represents the position of the $i$-singleton; $\mu_{i}(k-1)$ the activation of the rule at the previous instant; $\gamma(d e(k), r s(k))$ is the reward in accordance with the current values of $d e$ and $r s$.

The maximum displacement of the singletons was limited to \pm 0.2 with respect to the original value in the base controller. Moreover, the maximum displacement per cycle is limited to \pm 0.05 with respect to the previous position.

\subsection{Lateral Control}

Although a separation was made between the lateral and longitudinal controls, this does not mean that the actions on the steering wheel should not take the longitudinal state of the vehicle into account. On the contrary, several workers have taken the vehicle's speed as an input variable for the steering wheel control. For example, in (Lauer 2011) the speed is included as part of the state vector in the implementation of a reinforcement learning controller. Likewise, in (Pérez et al. 2011b) the maximum value of the turning rate of the steering wheel is set in accordance with the vehicle's speed and the distance to the nearest reference bend.

For this application, the vehicle's speed is considered to be a safety variable limiting both the maximum turn and the turn rate of the steering wheel. For this purpose, a dual controller was implemented. The idea is to cluster all the possible scenarios into two main use cases, so that two controllers are necessary:

- a low-speed controller, mostly for urban scenarios where the presence of sharp turns requires a wide range of movement of the steering wheel;

- a high-speed controller, for scenarios in which the car travels at over $50 \mathrm{~km} / \mathrm{h}$, requiring smoother movements of the steering wheel with a reduced range.

As a single output is needed, the controllers' outputs are merged proportionally to the vehicle's speed.

For the implementation of the two controllers, the same input-output configuration is used: two input variables - the vehicle's angular and lateral errors - and a sin- 
gle output - the reference position for the steering wheel. The angular error is defined as the angle between the vehicle's longitudinal axis and the reference path, measured in degrees. The lateral error is the deviation of the front of the vehicle from the reference, measured in metres. These variables are presented graphically in Fig. 5. The output is codified by means of singletons distributed in the $[-1,1]$ range, with -1 being the maximum possible turn of the steering wheel to the right and 1 the maximum possible turn to the left -540 degrees for each side.

The application of the lateral control configuration in previous works of the AUTOPIA program was conceived as a hybrid control system with two driving states: straight mode and curve mode (Naranjo et al. 2005). In the straight mode, the controller output was limited, so that large adjustments were avoided. In the curve mode, no restrictions were applied. This was necessary to improve the vehicle's stability on straight segments without affecting the performance on curves, where a wide turn range is necessary. Moreover, the steering wheel presented slight oscillations on scenarios with long bends - e.g., roundabouts. These oscillations were caused by the correction of both the lateral and the angular errors when the vehicle was still turning, meaning that the reference position changed to a value near zero, and the vehicle went in a straight line until the error was large enough to be corrected again. To solve this issue, a feedforward term has been added to the steering control expression. The main goal is to anticipate as much as possible the necessary steering angle to properly negotiate the trajectory bend, meaning the controller does not make big adjustments but smaller ones in accordance with the lateral and angular errors. This will improve the vehicle control in urban environments without including any driving mode.

For each trajectory segment, the bend is estimated by approximating the trajectory to a circumference. This is done by using three points: the start and end of the present segment and the start of the previous one. Once the bend is known, the open-loop steering angle is calculated in accordance with:

$$
\delta_{0}=\alpha \arctan (W B),
$$

where: $B$ is the trajectory bend; $W$ is the wheelbase of the vehicle - i.e., the distance between the front and rear axles; $\alpha$ is the steering ratio between the steering wheel and the front wheels.

Fig. 6 shows the MFs defined for each controller. It can be seen that, for the low-speed case, the MFs for both inputs are wider than for the high-speed case. This is because in urban scenarios the errors reach greater values without the vehicle being off the trajectory - e.g.,

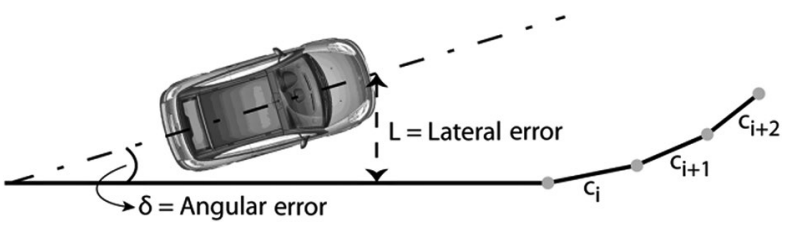

Fig. 5. Input variables defined for lateral control a)
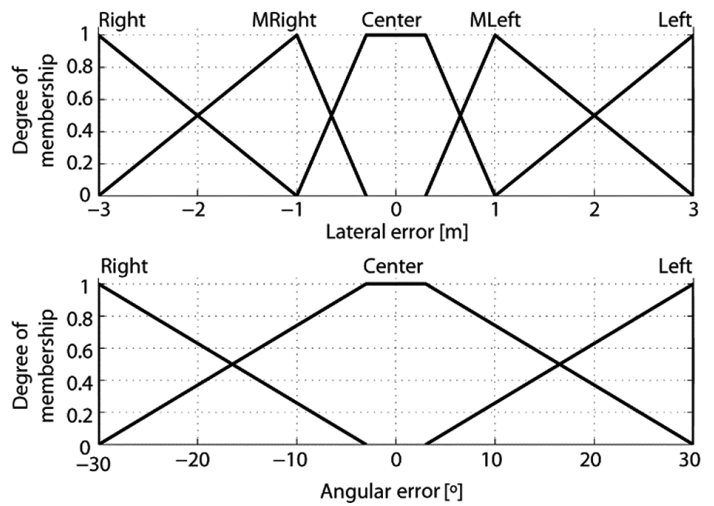

b)
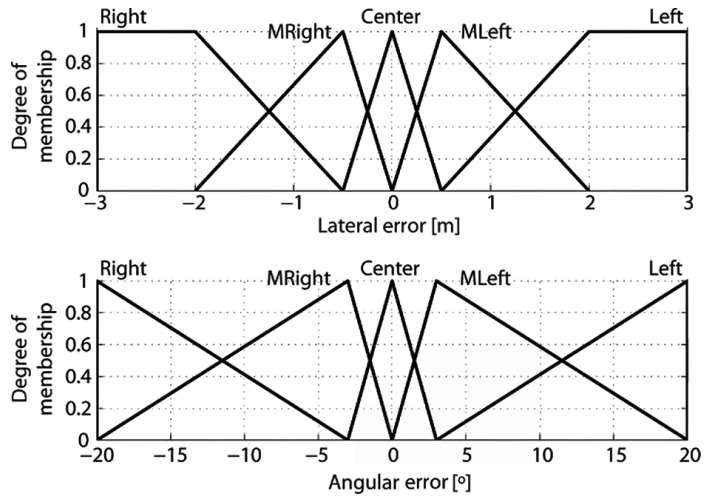

Fig. 6. Membership functions for lateral control: a - low-speed controller; b - high-speed controller

in sharp turns. For the high-speed case, the angular error is codified using two additional MFs.

The output surfaces for low-speed and high-speed controllers are shown in Fig. 7. It can be appreciated that, for the low-speed case, the surface evolves more smoothly than for the high-speed controller, with values in the range \pm 0.3 only. This is because the MFs defined for the inputs are broader. The high-speed case demands a faster response to changes in inputs since the stability of the vehicle decreases as the speed increases.

Despite having a wide output range, the high-speed controller is limited externally to improve the safety of the system. On the one hand, sudden changes of the steering wheel reference must be avoided. And on the other, the maximum turn of the wheels must be limited in accordance with the vehicle's speed to avoid it drifting. To achieve the first goal, the system limits the maximum change of the steering position to \pm 0.066 per cycle, which corresponds to one entire turn of the steering wheel per second. For the second goal, the maximum position of the steering wheel was limited to \pm 0.5 when the vehicle is traveling slower than $30 \mathrm{~km} / \mathrm{h}$ and \pm 0.2 turns when the vehicle is traveling faster than $80 \mathrm{~km} / \mathrm{h}$, decreasing proportionally to the vehicle's speed in the range between these two speed thresholds. These values were obtained by comparing the amplitude of the steering movements of different human drivers at different speeds.

Finally, the reference for the steering wheel $\delta_{S T}$ is estimated by merging the reference outputs for the low- 
a)

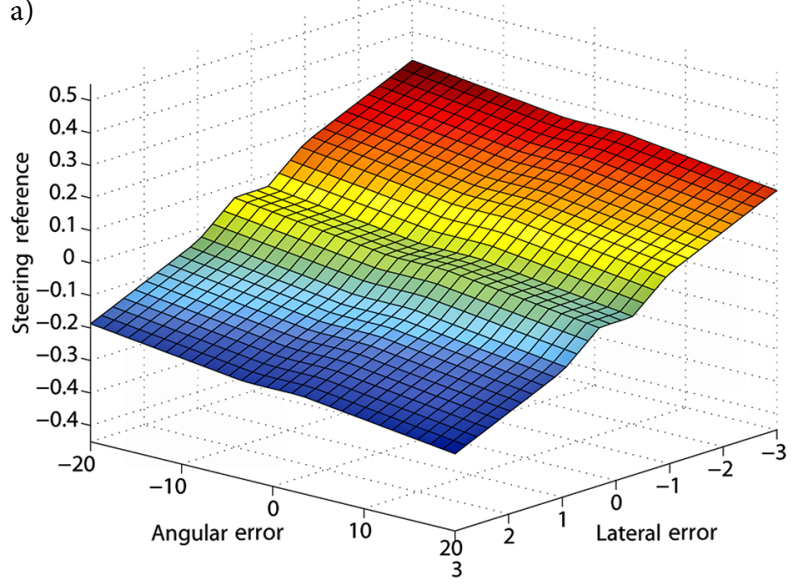

b)

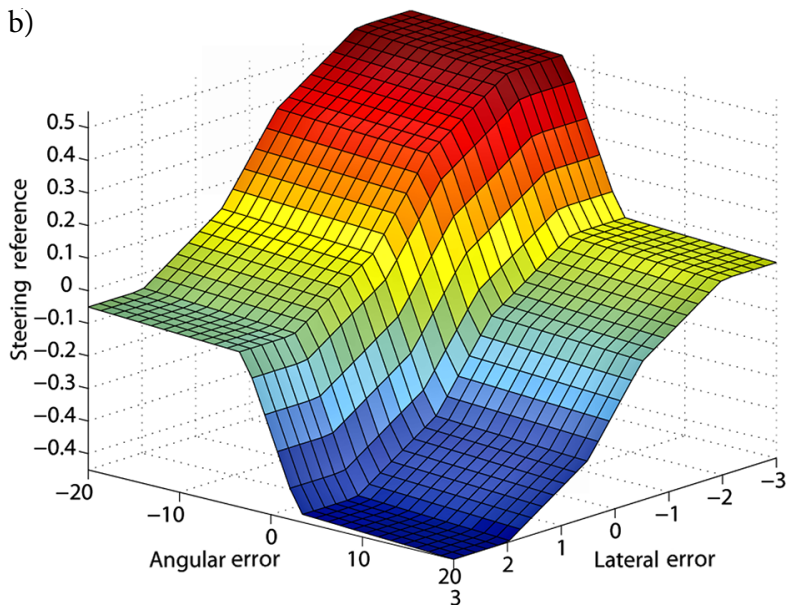

Fig. 7. Control surfaces for lateral control: $\mathrm{a}$ - low-speed controller; $\mathrm{b}$ - high-speed controller

speed and high-speed cases $\left(\delta_{S L}\right.$ and $\delta_{S H}$, respectively) in accordance with the vehicle's speed $v_{f}$ :

$$
\delta_{S T}= \begin{cases}\delta_{S L}, & \text { if } v_{f} \in[0,20) ; \\ \delta_{S L} \frac{50-v_{f}}{30}+\delta_{S H} \frac{v_{f}-20}{30}, & \text { if } v_{f} \in[20,50) \\ \delta_{S H}, & \text { if } v_{f} \in[50, \infty)\end{cases}
$$

with $20 \mathrm{~km} / \mathrm{h}$ being the maximum speed for the lowspeed controller, and $50 \mathrm{~km} / \mathrm{h}$ the minimum speed for the high-speed case.

\section{Results}

With the goal of validating the performance of the system, a public demonstration was conducted on June 2012. In contrast with previous works tested on private circuits, this demonstration was conducted on public roads around Madrid, Spain. Two Citroën C3 vehicles were used in the test: Clavileño as leading vehicle and Platero as trailing vehicle. Both vehicles are equipped with the AUTOPIA control architecture described in Section 1.

The route - shown in Fig. 8 - was selected for the total length to be around $100 \mathrm{~km}$, including both urban and motorway sections. It started from San Lorenzo

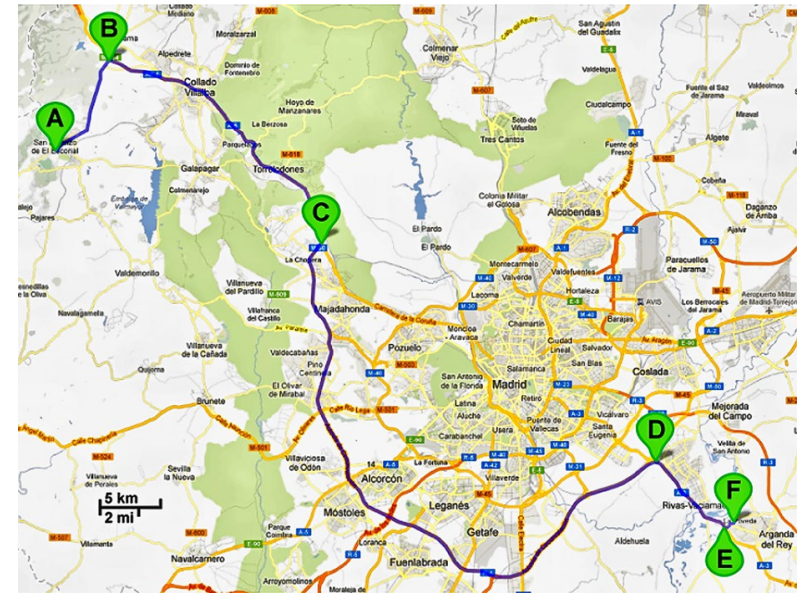

Fig. 8. Route performed by the autonomous vehicles between San Lorenzo de El Escorial and the Centre for Automation and Robotics in Arganda del Rey (image source: Google Maps)

de El Escorial - a town located $45 \mathrm{~km}$ northwest of Madrid - and ended at the facilities of the CAR in Arganda del Rey - around $22 \mathrm{~km}$ southeast of Madrid. Six checkpoints are marked on the route indicating:

- $A$ : the route's start at San Lorenzo de El Escorial;

- B: the entrance to the A6 motorway. End of the first segment of urban roads - at about $9.5 \mathrm{~km}$;

- $C$ : the exit from the A6 onto the M50 orbital motorway: at about $25 \mathrm{~km}$ from $B$;

- $D$ : the exit from the M50 onto the A3 motorway: at about $54 \mathrm{~km}$ from $C$;

- E: the exit from the A3 motorway onto the last segment of urban roads: at about $8 \mathrm{~km}$ from $D$;

- F: the route's end at the CAR facilities in Arganda del Rey: $1.3 \mathrm{~km}$ of urban roads.

Therefore the route traverses $11 \%$ of its length on urban roads and $89 \%$ on motorways environment.

The demonstration was organized in collaboration with the city council of San Lorenzo de El Escorial, the General Directorate of Traffic (DGT), and the highway patrol of the Civil Guard. The participation of these entities was fundamental for the experiment since the Spanish regulation on traffic does not authorize the circulation of autonomous vehicles on roads. For this reason, the two vehicles were treated by the authorities in accordance with the regulations for special transport, being escorted by traffic units throughout the test. Fig. 9 shows two pictures taken during the demonstration. The Fig. 9a shows both the leading and the trailing vehicles on the motorway. The Fig. $9 \mathrm{~b}$ shows a scene inside the trailing vehicle with the human driver reading a book while the car drives itself.

Fig. 10 depicts the vehicles' trajectory between the 6th and 9th minutes of the test. This corresponds to the first urban segment - i.e., between points A and B. Fig. 11 shows the evolution of the speed of the two vehicles. One sees that the minimum values of the speed (around $30 \mathrm{~km} / \mathrm{h}$ ) correspond to the two roundabouts present on the route (minutes 6.5 and 7.9 approximately). For the straight segments, the speed reached values of around $50 \mathrm{~km} / \mathrm{h}$. 
a)

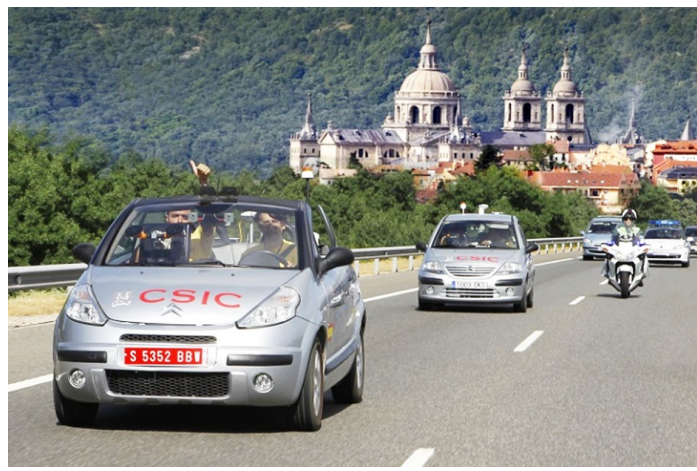

b)

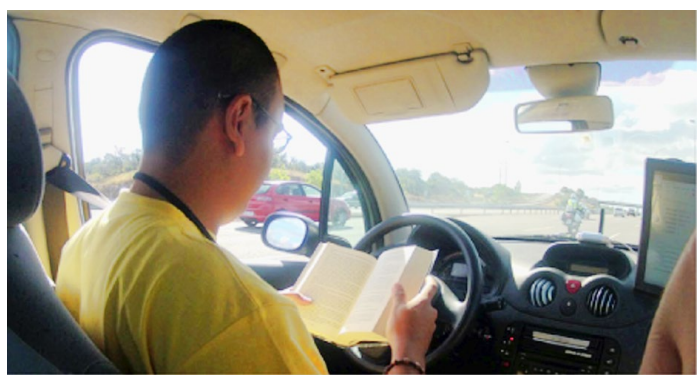

Fig. 9. Demonstration on the motorway: a - Clavileño and Platero during the experiment; $\mathrm{b}$ - inside the trailing vehicle, showing the 'driver' reading a book

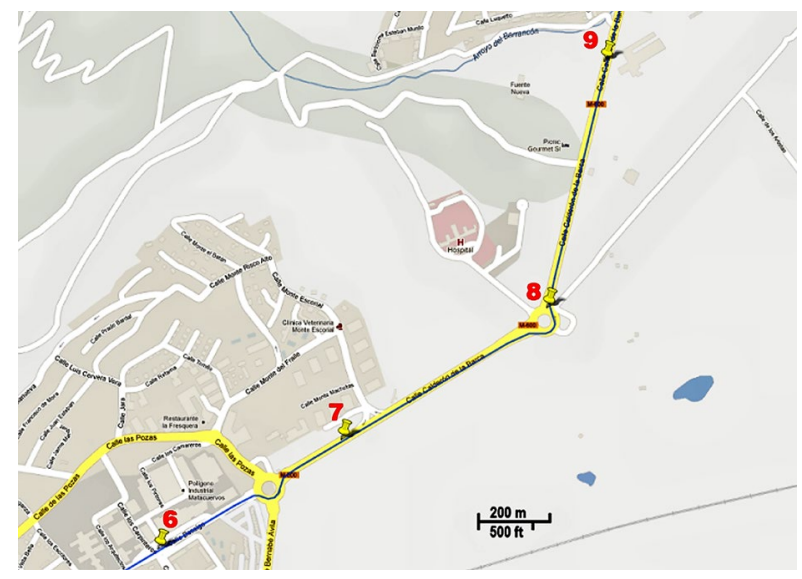

Fig. 10. Urban route between the 6th and 9th minutes, segment between points A and B (image source: Google Maps)

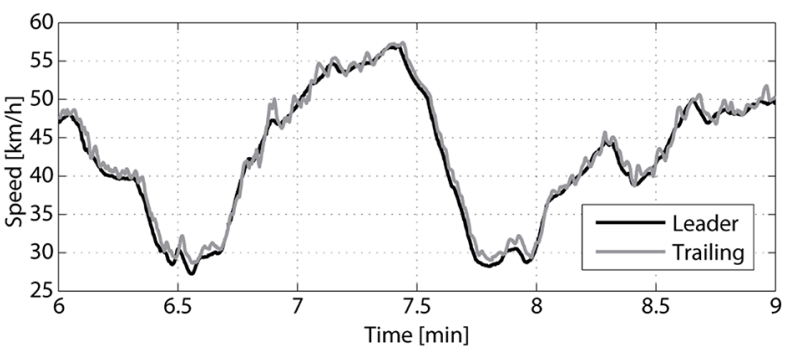

Fig. 11. Speed of the two vehicles in the urban area

The evolution of the two input variables for longitudinal control is shown in Fig. 12. It can be appreciated that the trailing vehicle was able to follow the distance reference with only a \pm 1 metre error. The relative speed between the vehicles always remained within the range $\pm 3 \mathrm{~km} / \mathrm{h}$. As shown in Fig. 13, the controller output took positive values in general. This means that the control action was mostly applied to the throttle rather than to the brake. This is relevant from the point of view of efficiency since every time the vehicle brakes, it wastes energy.

Regarding the lateral control, the two inputs are shown in Fig. 14. As was the case for the longitudinal control, the passage of the vehicle through the roundabouts is easily identified at around minutes 6.5 and 7.9. At each roundabout entrance, both the angular and the lateral errors increase, and indicate that the reference trajectory goes to the right of the current position of the vehicle's front. Once inside the roundabout, the errors take negative values due to the leftward curvature of the trajectory. Finally, the values are positive again for the roundabout's exit. For the straight segments, it can be appreciated that the lateral error remains within the range \pm 0.5 metres, while the angular error is within the range \pm 4 degrees.

As was mentioned in Section 4.2, the lateral error is measured from the front of the vehicle to the reference, thus it does not truly show the performance regarding the trajectory tracking. Therefore, a comparison between the real paths of both vehicles - measured on the middle point of the rear axis - is presented on Fig. 15 .

a)

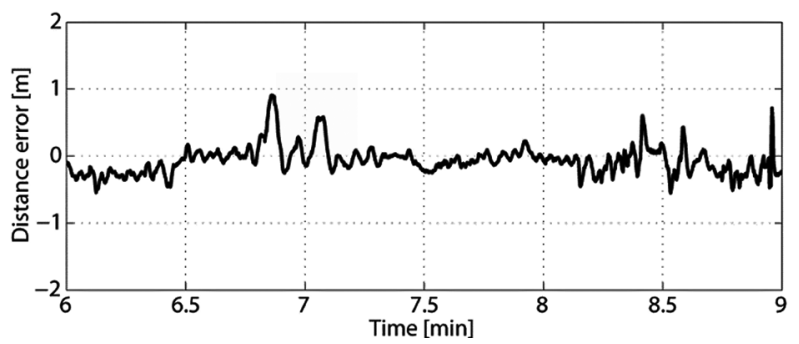

b)

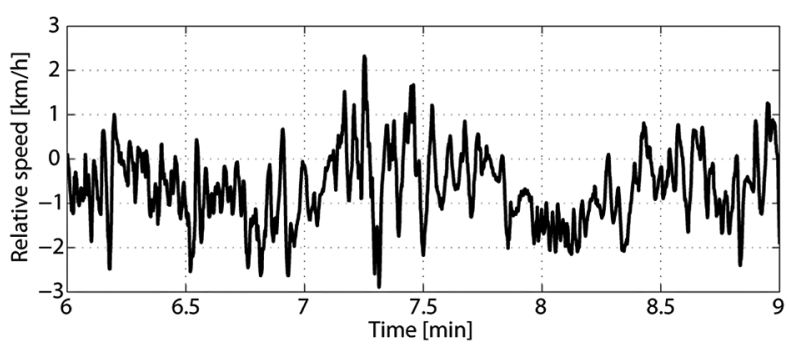

Fig. 12. Evolution of the input variables for longitudinal control: $\mathrm{a}$ - distance error; $\mathrm{b}$ - relative speed

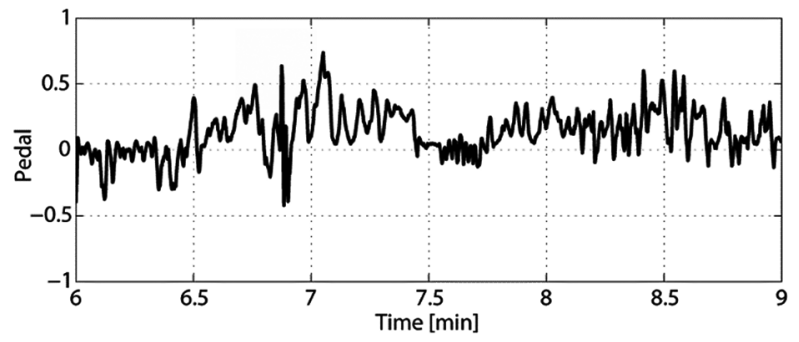

Fig. 13. Evolution of the output variable for longitudinal control 
a)

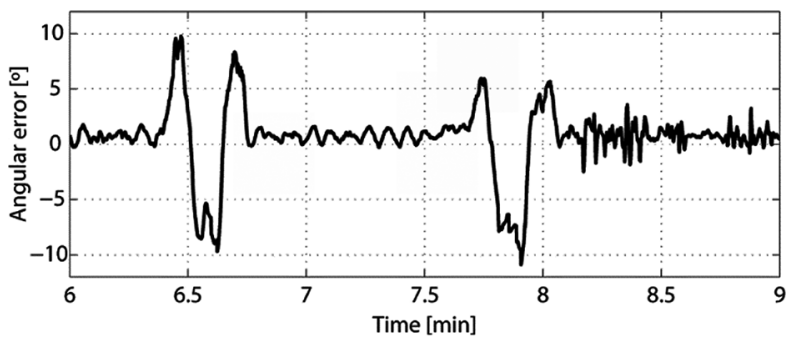

b)

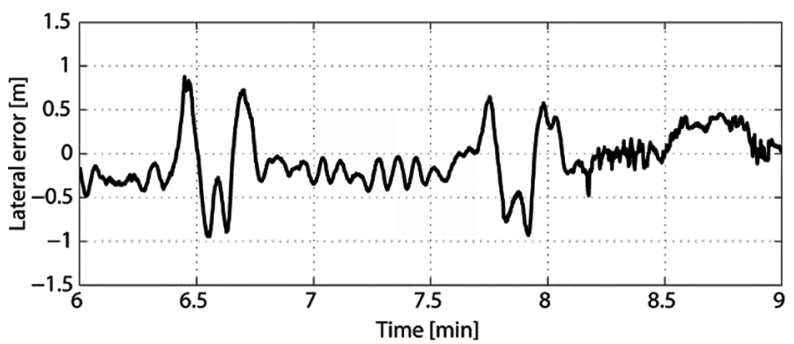

Fig. 14. Evolution of the input variables for lateral control: $a$ - angular error; $b$ - lateral error

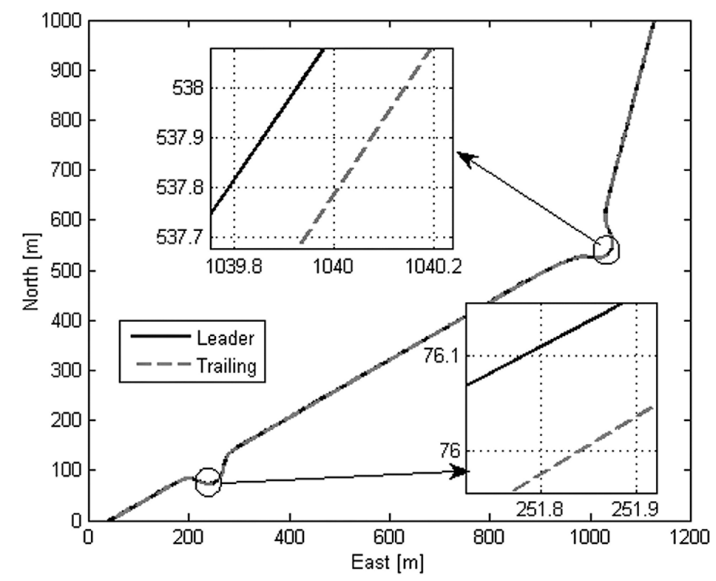

Fig. 15. Trajectory of the vehicles on urban environment

One can appreciate that the difference between the paths is considerably lower than the value of the lateral error, remaining lower than $25 \mathrm{~cm}$.

Fig. 16 shows the control output responding adequately to the inputs. For the straight segments, the reference remains at around zero with small variations. Then, in order to drive the vehicle through the roundabouts, the reference indicates right-left-right turns on the steering wheel.

Similar results for the behaviour of the trailing vehicle were found when the vehicles were traveling on the motorway. With the leader's speed increasing from 70 to $80 \mathrm{~km} / \mathrm{h}$ (Fig. 17) the trailing vehicle was able to follow the distance reference with an error within the range \pm 1 metres - image in Fig. 18a. One sees that the error value remains mostly around zero except for the three peaks present. These peaks correspond to sudden accelerations of the leading vehicle, as can also be appreciated in the evolution of the relative speed - image in Fig. 18b.

For the lateral control, it was found that the range of the two input errors - shown in Fig. 19 - is narrower

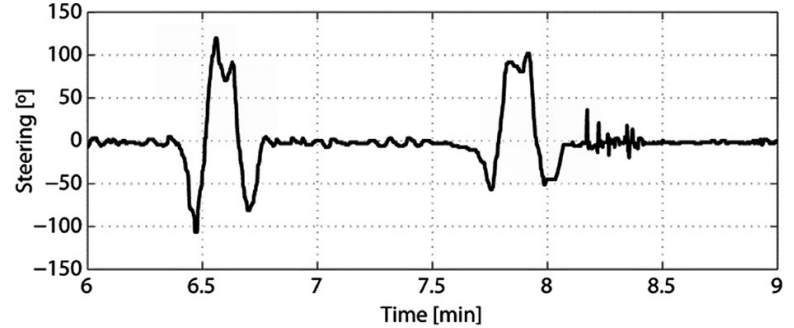

Fig. 16. Evolution of the output for lateral control

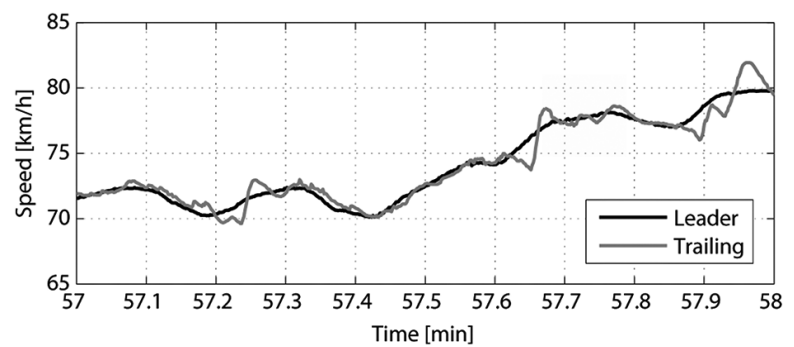

Fig. 17. Speed of the two vehicles on the motorway
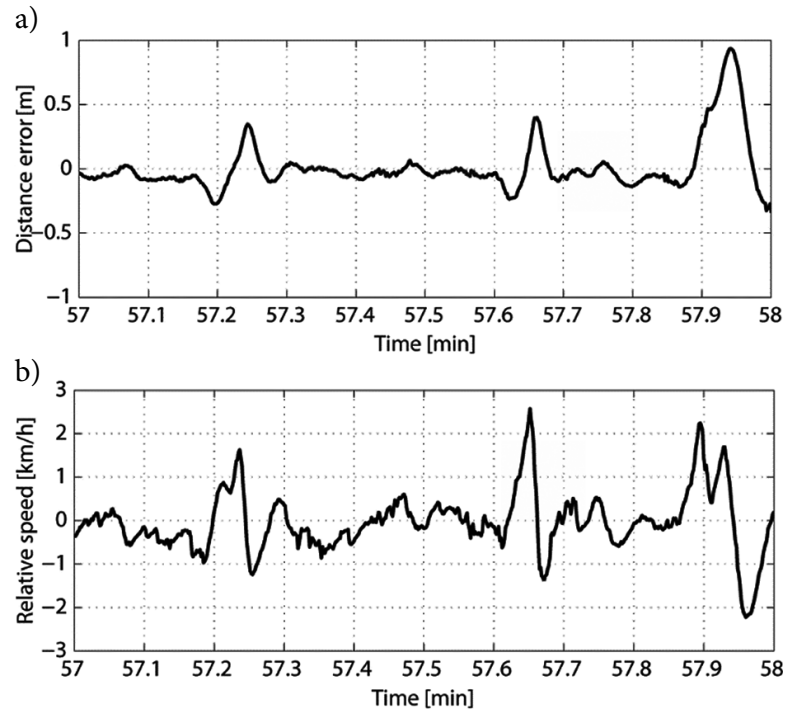

Fig. 18. Evolution of the input variables for longitudinal control in the motorway scenario: $\mathrm{a}$ - distance error; $\mathrm{b}$ - relative speed

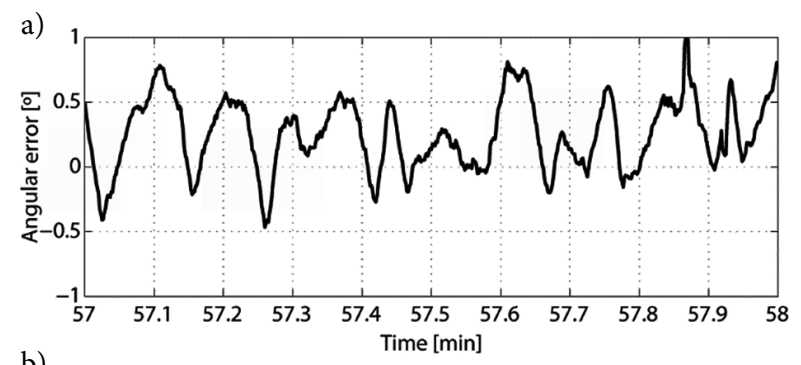

b)

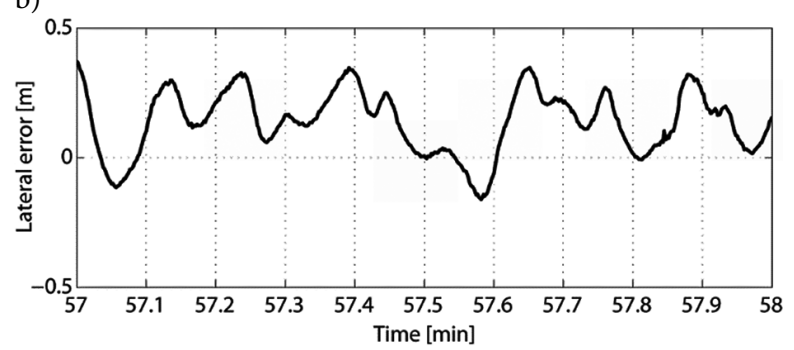

Fig. 19. Evolution of the input variables for lateral control: $\mathrm{a}$ - angular error; $\mathrm{b}$ - lateral error 
than for the previous scenario with the roundabouts. This seems natural since the expected curvature for the vehicle's trajectory when traveling over the motorway is considerably less than when traveling in urban environments.

\section{Conclusions and Future Work}

This communication has presented a system for autonomous driving on open roads. The proposal is based on inter-vehicle cooperation, where a leading vehicle shares information about its route in real time. Thanks to this information, a trailing vehicle is able to follow the leader trajectory using a combination of fuzzy logic controllers. Moreover, it is also able to maintain a reference time gap with the leader by implementing a CACC system. Compared to the closest tests developed up to now, the main contributions of this work to the state-of-the-art are:

- to the best of our knowledge, this is one of the first communications fully describing the control system and techniques required to perform an experiment with autonomous vehicles on open roads;

- it introduces a different control approach for controlling autonomous vehicles on urban and motorway environments;

- a method for online adjustment of the CACC fuzzy controller is described and implemented, coping with the most relevant disturbances and uncertain parameters, such as road slopes, passenger weight, or gear ratio;

- the experiment successfully proves the capability of the developed system to drive more than one hundred kilometres autonomously.

A public demonstration of the described system was conducted in June 2012, comprising a 100-km route through urban and motorway environments. As a strong point, the tracking results obtained with the CACC system were very precise, with the distance error being kept to less than 1 metre. Likewise, the lateral control was able to maintain the vehicle on the path of the leader with acceptable errors for both scenarios. As a weak point, the localization system needs to be improved to allow longer GPS gaps. Although the present system was able to cope with such gaps as motorway overpasses, traffic signals, etc., the presence of a 900-metre-long tunnel on the $\mathrm{M}-50$ forced the deactivation of the autonomous system while the vehicle passed through.

As future work, new sensors and filtering methods for data fusion will be included, improving the system capability to overcome scenarios where the GPS signal is strongly affected by long outages. Moreover, a second experiment is planned for evaluating the system performance with more vehicles, even including nonautonomous vehicles and different sensors on board. In this sense, the test will incorporate autonomous, semiautonomous, and human-driven vehicles at the same time, comparing their performance in tracking a leading vehicle in a platoon formation. On the other hand, the performance of the vehicles on urban environments must be improved for common manoeuvres. To that end, the cooperative controllers previously developed for overtaking, intersections and roundabouts will be integrated within this system.

\section{Acknowledgements}

This work was supported by the Spanish Ministry of Science and Innovation by means of Research Grants TRA2011-27712-C02-01 and TRA2011-14112-E.

Jorge Godoy wants to especially thank the JAE program (Spanish National Research Council - CSIC) for its support in the development of this work.

\section{References}

Bergenhem, C.; Huang, Q.; Benmimoun, A.; Robinson, T. 2010. Challenges of platooning on public motorways, in Proceedings of the 17th ITS World Congress, 25-29 October 2010, Busan, Korea, 1-12.

Bertozzi, M.; Broggi, A.; Coati, A.; Fedriga, R. I. 2013. A $13,000 \mathrm{~km}$ intercontinental trip with driverless vehicles: the VIAC experiment, IEEE Intelligent Transportation Systems Magazine 5(1): 28-41.

http://dx.doi.org/10.1109/MITS.2012.2225651

Car2Car. 2012. Car 2 Car Communication Consortium. Available from Internet: http://www.car-to-car.org

De Jongh, J. 2011. GCDC Communications Stack - Building and Installing the CALM FAST Router. Available from Internet: http://www.gcdc.net

Distner, M.; Bengtsson, M.; Broberg, T.; Jakobsson, L. 2009. City safety - a system addressing rear-end collisions at low speeds, in Proceedings of the 21st International Technical Conference on the Enhanced Safety of Vehicles (ESV), 15-18 June, Stuttgart, Germany. Paper No 09-0371, 1-7. Available from Internet: http://www-nrd.nhtsa.dot.gov/pdf/esv/ esv21/09-0371.pdf

Durrant-Whyte, H.; Bailey, T. 2006. Simultaneous localization and mapping: part I, IEEE Robotics \& Automation Magazine 13(2): 99-110. http://dx.doi.org/10.1109/MRA.2006.1638022

Eurostat. 2012. European Commission. Available from Internet: http://epp.eurostat.ec.europa.eu

García Rosa, R.; De Pedro Lucio, T. 2000. First applications of the ORBEX coprocessor: control of unmanned vehicles, Mathware and Soft Computing 7(2-3): 265-273.

GCDC 2012. Grand Cooperative Driving Challenge. Available from Internet: http://www.gcdc.net

Godoy, J.; Gruyer, D.; Lambert, A.; Villagrá, J. 2012. Development of an particle swarm algorithm for vehicle localization, in Proceedings of the 2012 IEEE Intelligent Vehicles Symposium (IV), 3-7 June 2012, Alcalá de Henares, Spain, 1114-1119. http://dx.doi.org/10.1109/IVS.2012.6232213

Kwakkernaat, M. 2011. GCDC 2011 Rules and Technology Document. Available from Internet: http://www.gcdc.net

Lategahn, H.; Geiger, A.; Kitt, B. 2011. Visual SLAM for autonomous ground vehicles, in Proceedings of the 2011 IEEE International Conference on Robotics and Automation (ICRA), 9-13 May 2011, Shangai, China, 1732-1737. http://dx.doi.org/10.1109/ICRA.2011.5979711

Lauer, M. 2011. A case study on learning a steering controller from scratch with reinforcement learning, in Proceedings of the 2011 IEEE Intelligent Vehicles Symposium (IV), 5-9 June 2011, Baden-Baden, Germany, 260-265. http://dx.doi.org/10.1109/IVS.2011.5940478 
Levinson, J.; Askeland, J.; Becker, J.; Dolson, J.; Held, D.; Kammel, S.; Kolter, J. Z.; Langer, D.; Pink, O.; Pratt, V.; Sokolsky, M.; Stanek, G.; Stavens, D.; Teichman, A.; Werling, M.; Thrun, S. 2011. Towards fully autonomous driving: systems and algorithms, in Proceedings of the 2011 IEEE Intelligent Vehicles Symposium (IV), 5-9 June 2011, Baden-Baden, Germany, 163-168.

http://dx.doi.org/10.1109/IVS.2011.5940562

Milanés, V.; Godoy, J.; Villagrá, J.; Pérez, J. 2011a. Automated on-ramp merging system for congested traffic situations, IEEE Transactions on Intelligent Transportation Systems 12(2): 500-508.

http://dx.doi.org/10.1109/TITS.2010.2096812

Milanés, V.; Naranjo, J. E.; González, C.; Alonso, J.; De Pedro, T. 2008. Autonomous vehicle based in cooperative GPS and inertial systems, Robotica 26(05): 627-633. http://dx.doi.org/10.1017/S0263574708004232

Milanés, V.; Onieva, E.; Pérez, J.; Simó, J.; González, C.; De Pedro, T. 2011b. Making transport safer: V2V-based automated emergency braking system, Transport 26(3): 290-302. http://dx.doi.org/10.3846/16484142.2011.622359

Milanés, V.; Shladover, S. E.; Spring, J.; Nowakowski, C.; Kawazoe, H.; Nakamura, M. 2014. Cooperative adaptive cruise control in real traffic situations, IEEE Transactions on Intelligent Transportation Systems 15(1): 296-305. http://dx.doi.org/10.1109/TITS.2013.2278494

Milanés, V.; Villagrá, J.; Godoy, J.; Simó, J.; Pérez, J.; Onieva, E. 2012. An intelligent V2I-based traffic management system, IEEE Transactions on Intelligent Transportation Systems 13(1): 49-58. http://dx.doi.org/10.1109/TITS.2011.2178839

Naranjo, J. E.; González, C.; García, R.; De Pedro, T.; Haber, R. E. 2005. Power-steering control architecture for automatic driving, IEEE Transactions on Intelligent Transportation Systems 6(4): 406-415. http://dx.doi.org/10.1109/TITS.2005.858622

Naranjo, J. E.; González, C.; García, R.; De Pedro, T. 2007. Cooperative throttle and brake fuzzy control for ACC+Stop\&Go maneuvers, IEEE Transactions on Vehicular Technology 56(4): 1623-1630. http://dx.doi.org/10.1109/TVT.2007.897632

Naranjo, J. E.; González, C.; García, R.; De Pedro, T. 2008. Lane-change fuzzy control in autonomous vehicles for the overtaking maneuver, IEEE Transactions on Intelligent Transportation Systems 9(3): 438-450. http://dx.doi.org/10.1109/TITS.2008.922880

Nowakowski, C.; O’Connell, J.; Shladover, S. E.; Cody, D. 2010. Cooperative adaptive cruise control: driver acceptance of following gap settings less than one second, in Proceedings of the Human Factors and Ergonomics Society Annual Meeting 54(24): 2033-2037.

http://dx.doi.org/10.1177/154193121005402403

Onieva, E.; Godoy, J.; Villagrá, J.; Milanés, V.; Pérez, J. 2013. Online learning of a fuzzy controller for a precise vehicle cruise control system, Expert Systems with Applications 40(4): 1046-1053. http://dx.doi.org/10.1016/j.eswa.2012.08.036

Onieva, E.; Milanés, V.; Pérez, J.; De Pedro, T. 2012. Genetic fuzzy-based steering wheel controller using a mass-produced car, International Journal of Innovative Computing, Information and Control 8(5B): 3477-3494.

Paz, L. M.; Tardos, J. D.; Neira, J. 2008. Divide and conquer: EKF SLAM in O(n), IEEE Transactions on Robotics 24(5): 1107-1120. http://dx.doi.org/10.1109/TRO.2008.2004639

Pérez, J.; Gajate, A.; Milanés, V.; Onieva, E.; Santos, M. 2010. Design and implementation of a neuro-fuzzy system for longitudinal control of autonomous vehicles, in Proceedings of the 2010 IEEE International Conference on Fuzzy Systems (FUZZ), 18-23 July 2010, Barcelona, Spain, 1-6. http://dx.doi.org/10.1109/FUZZY.2010.5584208

Pérez, J.; Milanés, V.; De Pedro, T.; Vlacic, L. 2011a. Autonomous driving manoeuvres in urban road traffic environment: a study on roundabouts, in Proceedings of the 18th IFAC World Congress, 28 August - 2 September 2011, Milano, Italy, 13795-138000. http://dx.doi.org/10.3182/20110828-6-IT-1002.00423

Pérez, J.; Milanés, V.; Onieva, E. 2011b. Cascade architecture for lateral control in autonomous vehicles, IEEE Transactions on Intelligent Transportation Systems 12(1): 73-82. http://dx.doi.org/10.1109/TITS.2010.2060722

Rituerto, A.; Puig, L.; Guerrero, J. J. 2010. Visual SLAM with an omnidirectional camera, in Proceedings of the 2010 20th International Conference on Pattern Recognition (ICPR), 23-26 August 2010, Istanbul, Turkey, 348-351. http://dx.doi.org/10.1109/ICPR.2010.94

SARTRE. 2012. The SARTRE Project. Available from Internet: http://www.sartre-project.eu

Shladover, S. E. 2009. Cooperative (rather than autonomous) vehicle-highway automation systems, IEEE Intelligent Transportation Systems Magazine 1(1): 10-19. http://dx.doi.org/10.1109/MITS.2009.932716

Sugeno, M. 1999. On stability of fuzzy systems expressed by fuzzy rules with singleton consequents, IEEE Transactions on Fuzzy Systems 7(2): 201-224.

http://dx.doi.org/10.1109/91.755401

Tan, H.-S.; Bougler, B. 2001. Vehicle Lateral Warning, Guidance and Control Based on Magnetic Markers: PATH Report of AHSRA Smart Cruise 21 Proving Tests. Working Paper UCB-ITS-PWP-2001-6. Institute of Transportation Studies, University of California, Berkeley. 71 p. Available from Internet: http://www.path.berkeley.edu/sites/default/files/ publications/PWP-2001-06.pdf

Van Nunen, E.; Kwakkernaat, R. J. A. E.; Ploeg, J.; Netten, B. D. 2012. Cooperative competition for future mobility, IEEE Transactions on Intelligent Transportation Systems 13(3): 1018-1025. http://dx.doi.org/10.1109/TITS.2012.2200475

Varaiya, P. 1993. Smart cars on smart roads: problems of control, IEEE Transactions on Automatic Control 38(2): 195207. http://dx.doi.org/10.1109/9.250509

Zadeh, L. A. 1996. Fuzzy logic = computing with words, IEEE Transactions on Fuzzy Systems 4(2): 103-111. http://dx.doi.org/10.1109/91.493904

Zadeh, L. A. 1965. Fuzzy sets, Information and Control 8(3): 338353. http://dx.doi.org/10.1016/S0019-9958(65)90241-X 